\title{
Context-US association as a determinant of long-term habituation in the crab Chasmagnathus
}

\author{
DANIEL TOMSIC, MARÍA E. PEDREIRA, ARTURO ROMANO, \\ GABRIELA HERMITTE, and HÉCTOR MALDONADO \\ University of Buenos Aires, Buenos Aires, Argentina
}

\begin{abstract}
An opaque screen moving overhead elicits an escape response in the crab Chasmagnathus that habituates for a long period after just a few presentations. A series of experiments was performed to determine whether the crab's long-term habituation (LTH) is mediated by an association between contextual cues and the eliciting stimulus. Chasmagnathus failed to exhibit LTH when a visual cue in the experimental environment was changed between training and testing. In addition, long-term exposure to the context in the absence of the eliciting stimulus impaired LTH, both when the exposure preceded the habituation training (latent inhibition) and when the exposure came after the training (extinction). Long exposure to the context alone prior to training also produced a decrease in responsiveness to the eliciting stimulus, which confirmed previous results. However, both effects of long exposure were only manifested when the crabs spent a period of time between exposure and testing out of the experimental context. The results of this paper are interpreted as supporting the view that Chasmagnathus LTH can be understood largely by Wagner's associative theory of habituation.
\end{abstract}

More than a decade ago, we began a systematic study of habituation of the escape response to a visual danger stimulus in the crab Chasmagnathus granulatus (for a review, see Maldonado, Romano, \& Tomsic, 1997). In nature, this crab is chased by gulls. In the laboratory, upon sudden presentation of a rectangular screen passing overhead, the crab responds with a running reaction in an attempt to escape. The response declines over trials, and, after a single training session of 15 trials $(1 \mathrm{~h})$, the decrement persists for a long period (i.e., for 5 days, at least; Pedreira, Dimant, Tomsic, Quesada-Allue, \& Maldonado, 1995). The response decrement has been shown to meet most of the parametric criteria of habituation (Brunner \& Maldonado, 1988), to be stimulus specific (Lozada, Romano, \& Maldonado, 1990; Rakitin, Tomsic, \& Maldonado, 1991; Romano, Lozada, \& Maldonado, 1991), to be frequency specific (Lozada, 1993; Pedreira, Romano, Tomsic, Lozada, \& Maldonado, 1998), and to be dependent on both age and the time of day (Pereyra, de la Iglesia, \& Maldonado, 1996; Tomsic, Dimant, \& Maldonado, 1996). The adap-

This work was supported by the Consejo Nacional de Investigaciones Cientificas y Técnicas de Argentina, Grant PID 216-89, and by the Universidad de Buenos Aires, Grant Ex-134. The authors are deeply indebted to Bernard G. Schreurs for valuable suggestions on the manuscript and to Angel Vidal for technical assistance. D. Tomsic is at the Laboratory of Adaptive Systems, NINDS, NIH, Bethesda, MD. Correspondence concerning this article should be addressed to $\mathrm{H}$. Maldonado, Laboratorio Fisiología Comportamiento, Depto. Biología, Pab 2. Ciudad Universitaria, (1428) Buenos Aires, Argentina (e-mail: hector @biolo.bg.fcen.uba.ar).

-Accepted by previous editor, Robert A. Rescorla tive value of this remarkable memory capacity of Chasmagnathus was studied in a comparison with that of Pachygrapsus - that is, by comparing the ability for longterm habituation (LTH) in two species that are close in phylogeny but disparate in ecology (Tomsic, Massoni, \& Maldonado, 1993).

Two series of experiments from our laboratory have demonstrated that Chasmagnathus is able to show longterm memorial representation of context. First, crabs tested $24 \mathrm{~h}$ after being exposed for $2 \mathrm{~h}$ to the testing environment showed levels of responding to the phasic stimulus lower than those of a nonpreexposed group (Pedreira, Dimant, \& Maldonado, 1996; Pedreira et al., 1995; Saraco \& Maldonado, 1995; Tomsic et al., 1993). This effect, which we called the contextual memory effect, has also been observed in similar experiments with rats-although, in that case, it was described as a sensitization effect of the new environment on the nonpreexposed animals (see, e.g., Hall \& Channell, 1985; Korn \& Moyer, 1966). Second, crabs tested in an environment that differed in several cues from the former training background failed to show LTH, which reveals context specificity (Tomsic et al., 1993). At this juncture, it seemed pertinent to address a controversial question in habituation theory: Does an association between context and a to-be-habituated stimulus underlie LTH in Chasmagnathus?

Early studies of habituation considered that the observed response decrement is dependent solely on the parameters of unconditioned stimulus (US) presentation (see, e.g., Groves \& Thompson, 1970). Further studies led to a broadening of this concept of habituation to include elements of the experimental situation, in addition to the 


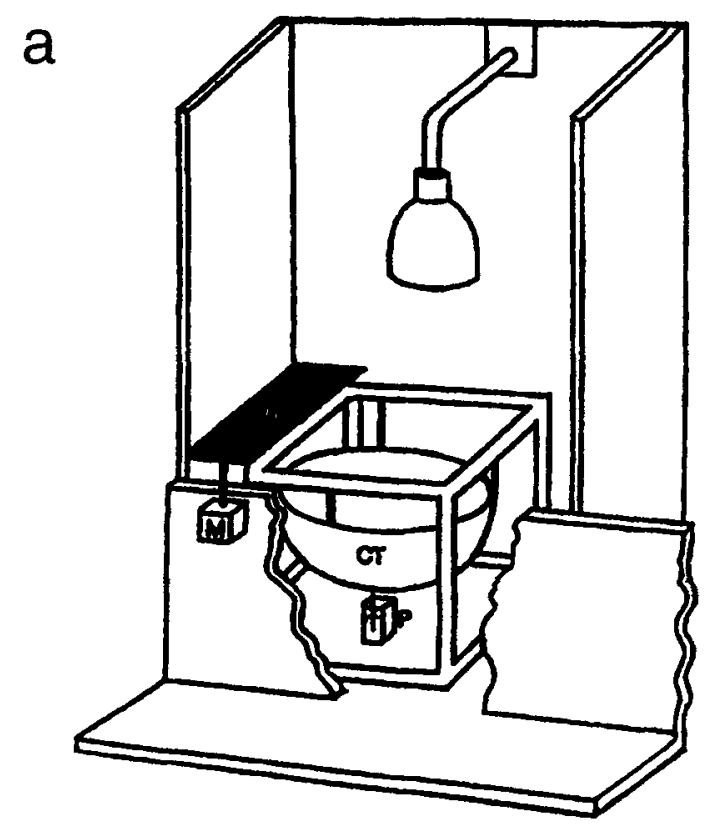

b
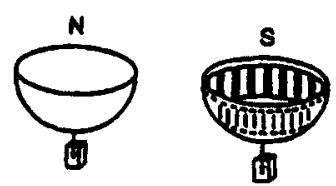

Figure 1. (a) The actometer, one of the 40 units of the apparatus. $C T$, plastic container; $R$, rectangular screen; $M$, motor; $P$, piezoelectric transducer. (b) $N$, normal context: the usual actometer container, as in (a); $S$, striped context: a ring $20 \mathrm{~cm}$ in diameter and $7 \mathrm{~cm}$ in height, with 2 -cm-wide black and white vertical strips, is located (Experiment 1) or hung (Experiment 2) inside the actometer container.

US, as critical determinants of responding. In particular, the US was now considered a complex of tonic and phasic cues, and response decrement to the US was considered to reflect habituation to the context plus habituation to the phasic stimulus (Korn \& Moyer, 1966; Marlin \& Miller, 1981). However, the increasing relevance of context to habituation reached its zenith in the associative theory of habituation (Wagner, 1976, 1978, 1979). Wagner distinguishes short- and LTH as being two processes subserved by different learning mechanisms. He argues that shortterm habituation depends solely on the repetition of US presentation. LTH, however, is postulated as resulting from associative processes. Specifically, he argues that animals form an association between the phasic stimulus and the context in which it is presented. Subsequent exposure to that context leads to a retrieval-generated representation of the target stimulus in short-term memory. The presence of this representation of the target stimulus in short-term memory dampens the animal's response to the actual physical stimulus - that is, the context becomes a conditioned stimulus (CS) (Whitlow \& Wagner, 1984).
Consequently, the present paper is aimed at further exploring the role of the context in LTH of Chasmagnathus, testing the main tenets of Wagner's associative theory of habituation-that is, context specificity and context-US contingency dependence.

\section{GENERAL METHOD}

\begin{abstract}
Animals
The animals were adult male Chasmagnathus granulatus crabs $2.7-2.9 \mathrm{~cm}$ across the carapace, collected from water less than $1 \mathrm{~m}$ deep in the rias (narrow coastal inlets) of San Clemente del Tuyú, Argentina, and transported to the laboratory, where they were lodged in plastic tanks $(35 \times 48 \times 27 \mathrm{~cm})$ filled to a depth of $2 \mathrm{~cm}$ with water, at a density of 35 crabs per tank. The water that was used in the tanks and other containers during the experiments was prepared with hw-Marinex (Winex-Germany) (salinity $1.0 \%-1.4 \%, \mathrm{pH} 7.4-7.6$ ). The holding room was maintained on a 12 -h light:dark cycle (light on 0700-1900 h). The animals were fed rabbit pellets (Nutrients SA, Argentina) every 3 days, and, after feeding, the water was changed. The temperature of both the holding and the experimental rooms, as well as the alley between them, was maintained within a range of $19^{\circ}-24^{\circ} \mathrm{C}$. Experiments were carried out within the first week after the animals' arrival and between November and June (i.e., late spring, summer, and fall). Each crab was used only in one experiment.
\end{abstract}

\section{Apparatus}

The apparatus has been previously described in detail (Romano et al., 1991). Briefly, the experimental unit is the actometer (Figure 1a), consisting of a bowl-shaped plastic container with a steep concave wall and a circular central flat floor $10 \mathrm{~cm}$ in diameter, covered to a depth of $0.5 \mathrm{~cm}$ with water (the actometer container, CT). The crab was placed in the container, which was suspended by three strings from an upper wooden framework $(23 \times 23 \times 30 \mathrm{~cm})$ and illuminated by a $10-\mathrm{W}$ lamp placed $30 \mathrm{~cm}$ above the animal. An opaque rectangle screen $(25 \times 7.5 \mathrm{~cm})(\mathrm{R})$ could be moved horizontally by a motor over the animal and across the upper surface of the framework in $2.3 \mathrm{sec}$. Screen displacements provoked a crab's running response and, consequently, container oscillations. A stylus was centrally cemented to the bottom of the container and connected to a piezoelectric transducer (P). Container oscillations induced, through the transducer, electrical signals proportional to the velocity of the oscillations. Such signals were amplified, integrated during the recording time $(9 \mathrm{sec})$, and translated into numerical units ranging from 0 to 1,530 , before being processed by the computer. Thus, the scores were correlated proportionally to the velocity and the number of the container oscillations recorded during $9 \mathrm{sec}$. The experimental room had 40 actometers, isolated from each other by partitions. A computer was employed to program trial sequences, trial duration, and intertrial intervals, as well as to monitor experimental events.

The escape response in the actometer consists of the crab starting to run, in an attempt to move away from the passing screen. However, because the steep concavity of the circular wall prevents the animal from climbing up, each running effort is confined to the flat center of the container, in such a way that the escape response during a single trial resembles a series of flights from the center toward the base of the wall.

\section{Experimental Procedure}

A stimulation session had a fixed number of trials given with 180sec intertrial intervals and preceded by $15 \mathrm{~min}$ of adaptation in the actometer. Each trial lasted $9 \mathrm{sec}$ and consisted of passing the screen four times over the actometer, recording the crab's activity during the entire trial time. Two sessions per experiment were run - that is, the training session ( 15 trials) and the testing session ( 1 trial), separated by a $24-\mathrm{h}$ intersession interval. During the intersession interval, 
crabs were individually lodged in plastic containers (the so-called rest containers) covered to a depth of $0.5 \mathrm{~cm}$ with water and kept inside drawers dimly lit, unless stated otherwise in the method section of each experiment.

The experimental design basically included two pairs of groups, each pair consisting of a trained group (T) that was given the 15 training trials and an untrained one $(U)$ that was kept in the actometers during the entire training session but without screen presentation. One trained-untrained pair (the standard pair) underwent the usual experimental conditions of a LTH experiment with Chasmagnathus, whereas the other pair was subjected to a treatment - that is, to a change in the usual experimental conditions (either a training-testing context shift or the addition of a long exposure to the context). Both pairs of groups received the same 1-trial testing session.

Before animals were assigned to an experiment, they underwent a selection test: Each crab was turned on its back, and only animals that immediately returned to their normal position were used. The rationale behind this selection is that crabs with a slow righting reaction show a low responsiveness to a large diversity of stimuli, and, at a later time, they usually present unhealthy symptoms. No more than $10 \%$ of tested crabs were discarded.

Since the number of actometers was not sufficient to run all animals in each experiment simultaneously, replications during the same day were necessary. An equal number of crabs per group was used in each replication, but animals of a same group were placed in different actometers each time. Thus, any potential effect of time of day and/or any between-actometer differences were offset.

The crab's baseline responsiveness to the passing screen proved to be consistent up to 10 days after arrival, but, on occasion, animals coming from different capture efforts presented differences in response level, a variability attributable to the different conditions in the ria at the moment of the capture. Therefore, only individuals belonging to the same capture were used in each experiment.

\section{Data Analysis and Evaluation of Memory Retention}

Long-term memory was assessed by focusing the data analysis on testing scores. Rescorla (1988) argued convincingly for the use of this sort of analysis instead of a paired training-testing comparison, stressing the need to distinguish clearly between time of input (training session) and time of assessment (testing session). This view is certainly justified in the present case, inasmuch as it has been demonstrated that LTH in the crab is independent of the escape response level at training (Tomsic, Maldonado, \& Rakitin, 1991), a result consistent with similar findings in other animals (Applewhite, Gardner, \& Lapan, 1969; Peeke \& Veno, 1976).

In all previous experiments at our laboratory, without exception, a significant difference ( $t$ test $=.05$ ) between the mean testing scores of a trained group (receiving 15 training trials with a 180 -sec intertrail interval) and that of its corresponding untrained group was shown $24 \mathrm{~h}$ after training. Therefore, a significant difference between groups of standard pairs was predicted for all of the experiments of this paper. In contrast, according to the hypothesis posited for each experiment, no significant difference between groups of the treated pairs was expected.

Test results were analyzed with a two-factor analysis of variance (ANOVA) and subsequent simple main effects analyses. The training performances of crabs that were being stimulated under different experimental conditions were evaluated by a repeated measures ANOVA. All statistical tests used a rejection criterion of $p<.05$.

\section{Definitions}

Throughout this paper, STH refers to the response decrement within the training session; LTH, to a retention of the response decrement shown in the testing session. Phasic stimulus stands for the iterated danger stimulus (i.e., US, target, or eliciting stimulus). A standard pair consists of one trained and one untrained group of crabs subjected to the same conditions in all the experiments. A treated pair, which receives specific denomination according to the experiment, consists also of one trained and one untrained group of crabs that was subjected to a treatment (i.e., to changes in the usual experimental conditions).

\section{EXPERIMENT 1}

Prior research from our laboratory (Tomsic et al., 1993) showed that Chasmagnathus LTH was wholly abolished when a multimodal change of context was made between sessions. The purpose of the present experiment was to investigate whether LTH was also impaired by changing only a visual contextual cue. In addition, the experiment contains a wider design than that previously adopted. In former research, the contextual incongruity between training and testing was unidirectional-that is, a context $\mathrm{A}$ was used at training and a context $B$ at testing but not vice versa - whereas, in the present design, a bidirectional incongruity was used.

\section{Method}

A ring $20 \mathrm{~cm}$ in diameter and $7 \mathrm{~cm}$ in height, with $2-\mathrm{cm}$-wide black and white vertical strips located inside the actometer container (Figure 1b), was used as the alternative environment and termed the striped context (S). The usual actometer container was considered to be the normal context $(\mathrm{N})$.

Two hundred sixty-four crabs were randomly assigned to eight groups of 33 crabs each that were distributed into four pairs: two standard pairs and two context-shift pairs. The trained groups of the standard pairs were trained and tested in the same context, either $\mathrm{N}$ or $\mathrm{S}$, whereas the trained groups of the context-shift pairs were either trained in S and tested in $\mathrm{N}$ or trained in $\mathrm{N}$ and tested in $\mathrm{S}$. Untrained groups were equal to their respective trained groups, except that they did not receive the phasic stimulation during the training session. Thus, crabs were distributed according to a $2 \times 2 \times 2$ factorial design, the factors being training (i.e., training or no training), context shift (i.e., same or different context at training and testing sessions), and testing context (i.e., testing in $\mathrm{N}$ or $\mathrm{S}$ context).

Should the hypothesis that LTH is context specific be correct, the context shift would be expected to disrupt memory retention, either in the group trained in $\mathrm{S}$ and tested in $\mathrm{N}$ or in that trained in $\mathrm{N}$ and tested in $\mathrm{S}$, without affecting the response level of untrained animals.

\section{Results and Discussion}

Two $2 \times 15$ ANOVAs of mixed repeated measures that were performed on training data revealed no significant difference between groups trained in the same context, so that training scores were pooled in two larger groups $(\mathrm{N}$ and S) of 66 crabs each. An ANOVA on these pooled data (Figure 2a) showed a significant difference between $\mathrm{N}$ and $S$ trained groups $[F(1,130)=9.5, p<.01]$ and a significant trial effect $[F(14,1820)=56.6, p<.01]$ but no significant context $\times$ trial interaction.

Figure $2 \mathrm{~b}$ presents the mean testing response score of the eight groups (left panel: groups tested in N; right panel: groups tested in S). A $2 \times 2 \times 2$ factorial ANOVA performed on these data showed a significant effect for the training factor $[F(1,256)=14.7, p<.01]$, for the context-shift factor $(F=6.2, p<.05)$, for the testing context $(F=5.2, p<.05)$, and marginal significant training $\times$ shift context interaction $(F=3.7, p \sim .05)$. No significant 

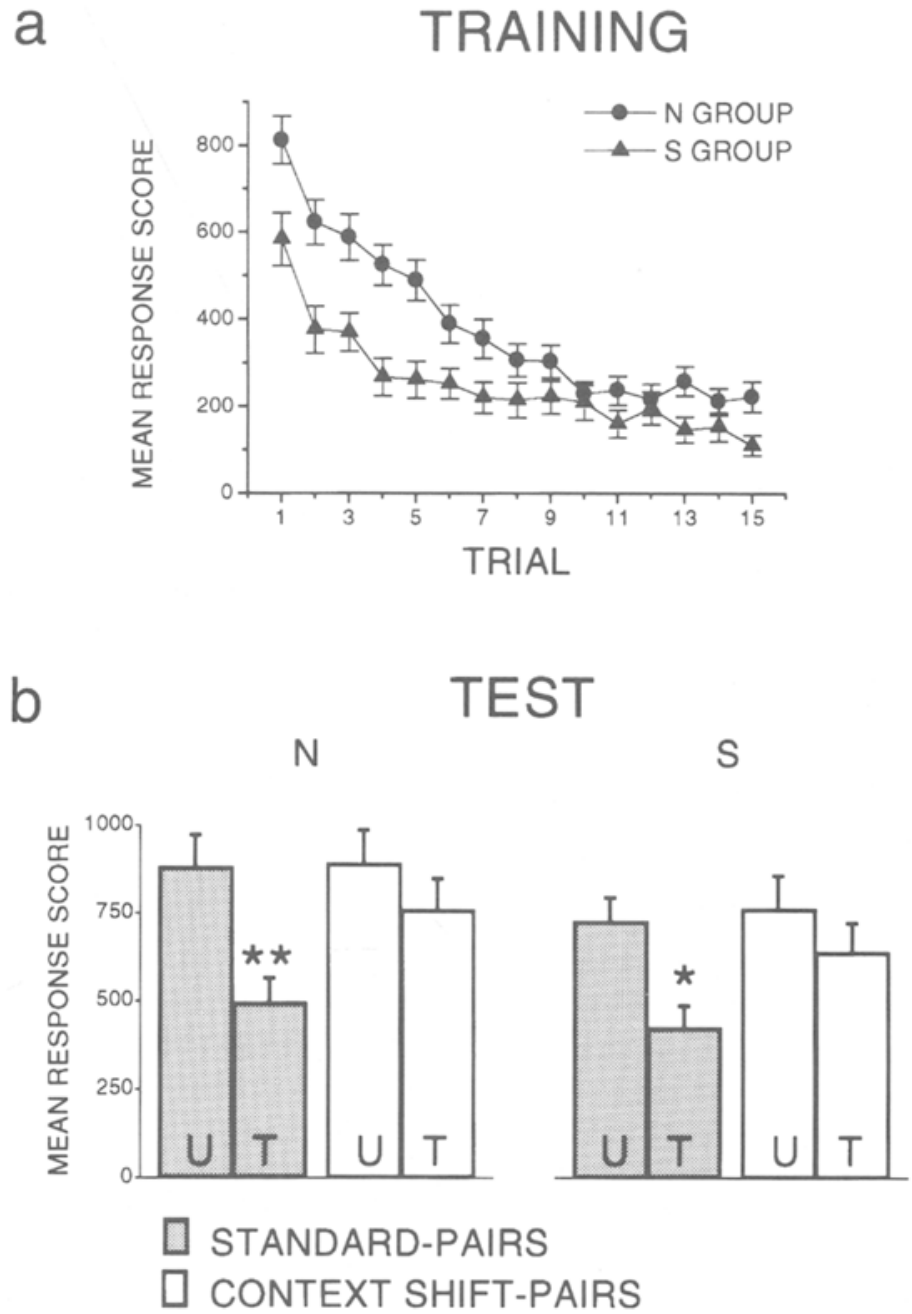

Figure 2. Unimodal change in the environment (Experiment 1): a striped ring placed inside the container actometer $(S)$ at training and a normal container ( $N$ ) at testing, or vice versa. (a) Training performance. Black circles stand for crabs trained in normal container $(N)$; black triangles, for crabs trained in striped container $(S)(n=66)$. Ordinate, mean response score $\pm S E M$. (b) Left side: Testing performance in normal container (N). Right side: Testing performance in striped container $(S)$. Gray bars: standard pair of untrained $(\mathrm{U})$ and trained $(\mathrm{T})$ groups with the same context at training and testing sessions. White bars: context-shift pair of untrained $(U)$ and trained $(T)$ groups with different context at training and testing sessions. Ordinate, mean response score $\pm S E M .{ }^{\star} p<.05 .{ }^{\star \star} p<.01$.

effect was disclosed for the other interactions. Subsequent simple main effects analyses showed a significant training effect for crabs trained and tested in $\mathrm{N}[F(1,256)=11.1$, $p<.01]$ and for those trained and tested in $\mathrm{S}(F=6.6, p<$ $.05)$. No significant effect was shown for crabs trained and tested in different contexts (i.e., either $\mathrm{N}-\mathrm{S}$ or $\mathrm{S}-\mathrm{N}$ ).

Therefore, according to the statistical analysis, it is reasonable to conclude that the contextual change affected LTH. Indeed, whichever the direction of the change, either from $\mathrm{N}$ to $\mathrm{S}$ or vice versa, a decrement in response habituation was found, whereas the usual retention was exhibited when both contexts were kept invariant. The lower response level of S groups at training (Figure 2a) is attributable to a shortening of the escape response caused by the striped ring in the actometer rather than by a disparity between unconditioned stimuli. Indeed, a visual inspection of the test performance also revealed a lower response level for the groups tested in the striped-ring context (Figure 2b) and, consequently, the factor ANOVA disclosed a significant test-context effect. However, this lower level of responsiveness can hardly explain the retention impairment shown by animals trained and tested in different con- 
texts. For example, we have previously found (Tomsic et al., 1991) that Chasmagnathus who had had their escape response completely abolished during training by the administration of GABA exhibited a LTH equivalent to that of crabs who actually performed the normal escape response during training. Thus, the escape response during training proved not to be necessary for the acquisition or retention of Chasmagnathus LTH. Taken together, this fact and the bidirectionality of the results obtained in the present experiment suggest that the shortening of the escape response does not explain the habituation impairment observed in the groups trained and tested in different contexts. However, the following experiment was aimed to completely rule out that possibility.

\section{EXPERIMENT 2}

In contrast with the previous experiment, the present one was designed to avoid any alteration of the escape response because of differences in the context when the animals are being trained.

\section{Method}

The same striped ring employed in the previous experiment, though now hung inside the actometer container without touching it, was used as the alternative environment and was termed the striped context $(\mathrm{S})$. The usual actometer container was considered to be the normal context $(\mathrm{N})$.

One hundred and sixty crabs were randomly assigned to four groups of 40 crabs each that were distributed into two pairs: the standard pair and the context-shift pair. The trained group of the standard pair was trained and tested in N, whereas the trained group of the treated pair was trained in $\mathrm{S}$ and tested in $\mathrm{N}$. Untrained groups were equal to their respective trained groups, except that they did not receive the phasic stimulation during the training session. Thus, the crabs were distributed according to a $2 \times 2$ factorial design, the factors being training (i.e., training or no training) and context shift (i.e., same or different context at training and testing sessions).

\section{Results and Discussion}

Two $2 \times 15$ ANOVAs of mixed repeated measures that were performed on training data (Figure $3 a$ ) revealed no significant difference between groups trained in the different context and no significant context $\times$ trial interaction but disclosed a significant trial effect $[F(14,1092)=38.2$, $p<.001]$.

Figure $3 \mathrm{~b}$ presents the mean testing response score. A factorial ANOVA that was performed on these data showed a significant effect for the training factor $[F(1,156)=7.7$, $p<.01]$ and a significant training $\times$ context-shift interaction $(F=4.1, p<.05)$ but no significant effect for the context-shift factor. Subsequent simple main effects analyses showed a significant training effect for crabs trained and tested in $N[F(1,156)=11.1, p<.01]$ but not for those trained in $\mathrm{S}$ and tested in $\mathrm{N}$, and no significant difference between untrained groups $(\mathrm{U})$, but a significant difference between trained groups $(\mathrm{T})[F(1,156)=4.8, p<.05]$.

Thus, the results of the present experiment confirm the fact that a contextual change produces an impairment in habituation. Moreover, because the two trained groups in this experiment displayed a similar level of response at training, we can exclude an interpretation for the results of Experiment 1 in terms of an artifact produced by the differences in performance. Taken together, the outcomes of this and the previous experiment represent new evidence that Chasmagnathus LTH is context specific. Thus, a prediction of Wagner's theory of habituation seems to be confirmed. However, dependency on environmental invariance is a necessary but not a sufficient condition. Indeed, views other than Wagner's theory also predict context specificity insofar as they consider the stimulus to be habituated as a complex stimulus that includes the contextual background (see, e.g., Marlin \& Miller, 1981). Therefore, the following experiments were aimed at testing other predictions of the associative theory by contrasting them with those of the nonassociative view.

\section{EXPERIMENT 3}

A corollary of the associative theory of habituation is that the lower the context-phasic stimulus contingency, the lower the retention of the habituated response. In order to test this corollary, the experimental design included not only the training session (contingent phase) but also periods during which animals remained in the actometer containers without presentation of the stimulus (noncontingent phases). In order to combine in a single experiment two effects that undermine context-US associations (one that interferes with acquisition of context-US association and one that extinguishes that learning), latent inhibition and extinction procedures were applied together. Therefore, two equal noncontingent phases of $12 \mathrm{~h}$ each were included, one before training and the other before testing. This design was also adopted in order to avoid differences between the periods that precede the training and the test, which otherwise could be held responsible for the lack of retention (Spear, 1973, 1978).

\section{Method}

The experimental design comprised the usual standard pair and a noncontingent pair. Four groups of 30 animals each included two trained groups (i.e., the standard and noncontingent trained groups) and the respective untrained ones (i.e., the standard and noncontingent untrained groups). Training and testing sessions were as usual for both trained groups. The experimental procedure for each untrained group was identical to that of its respective trained group, except that they were not presented the phasic stimulus during the training session. All groups passed through five phases (Figure $4 \mathrm{a}$ ): pretraining $(12 \mathrm{~h})$, training session $(1 \mathrm{~h})$, posttraining $(12 \mathrm{~h})$, pretesting $(12 \mathrm{~h})$, and testing session $(16 \mathrm{~min})$. The crabs of the standard groups were individually housed during either pretraining or pretesting in cylindrical containers that were different from the actometer containers, though with the same illumination, whereas those of the noncontingent groups expended such phases in the container actometers. During the posttraining phase, all groups were placed in the usual rest containers - that is, plastic bowls inside dimly lit drawers. Crabs in the noncontingent groups were handled 15 min before both the training session and the testing session in order to control for the necessary handling of the animals in the control groups when 


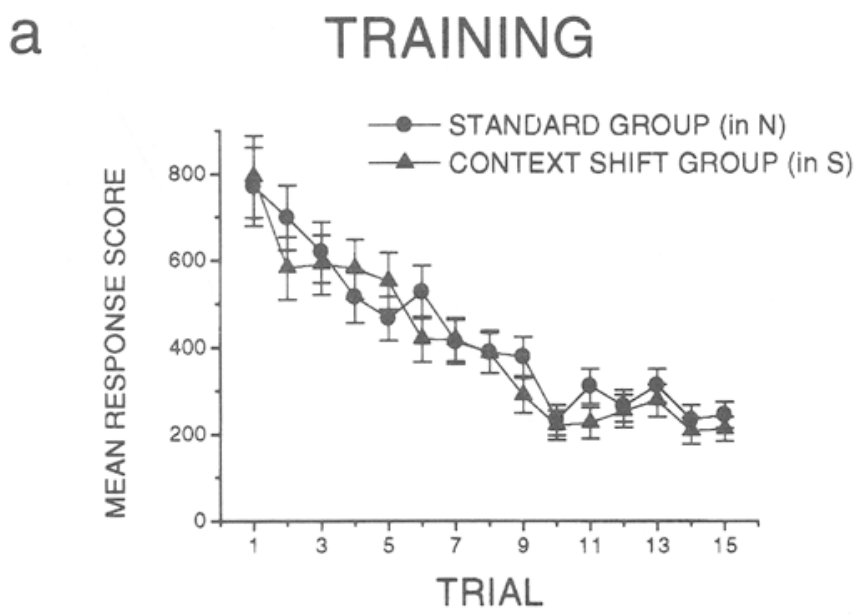

\section{b TEST IN NORMAL CONTAINERS (N)}

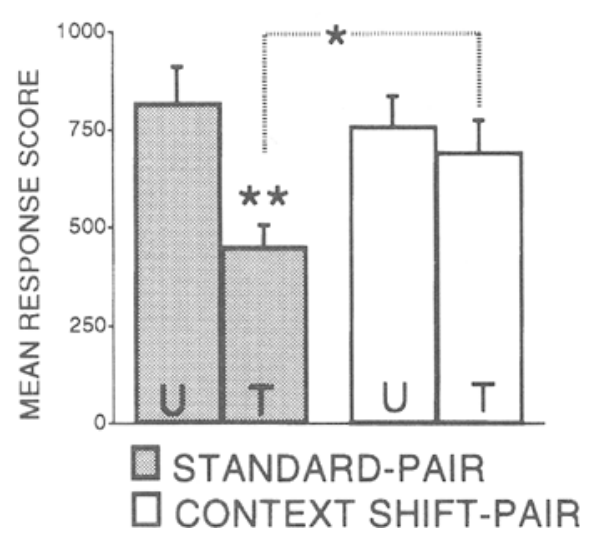

Figure 3. Unimodal change in the environment (Experiment 2): a striped ring hung inside the container actometer but without touching it $(S)$ at training and a normal container $(N)$ at testing. (a) Training performance. Symbols as in Figure $2 a(n=40)$. (b) Testing performance in normal container ( $\mathbf{N}$ ). Symbols as in Figure $2 \mathbf{b}$.

they were moved from the cylindrical containers to the actometer containers. Thus, the difference between standard and noncontingent groups resides in the fact that the latter, unlike the former, expend the pretraining and pretest phases in the actometers (* in Figure $4 \mathrm{a}$ ).

Previous experiments from our laboratory showed that a $2-\mathrm{h}$ preexposure to the context alone induces a reduction in the response level to the phasic stimulus when animals are tested $24 \mathrm{~h}$ later (i.e., the contextual memory effect; Pedreira et al., 1996, Pedreira et al., 1995). In consideration of this fact and in accordance with the associative hypothesis of habituation, it should be expected that the two periods of 12-h exposure to the context alone produce a double opposite effect on the response level at test-namely, a reduction in both noncontingent groups (untrained and trained) because of the contextual memory effect and a blockade of the reduction induced by training in the noncontingent-trained group because of a latent inhibition and/or extinction effect. Explicitly, it should be expected, first, that there will be a response level at test of the noncontingent-untrained group that is lower than that of the standard-untrained one, and, second, that there will be no difference between noncontingent groups. In contrast, if the nonassociative model of habituation is adopted (see, e.g., Korn \& Moyer, 1966; Marlin \& Miller, 1981), the response level of the noncontingent-trained group is expected to be lower than that of the noncontingent-untrained group, because its performance is assumed to be under the impact of both the contextual memory effect and LTH.

\section{Results and Discussion}

Figure $4 \mathrm{~b}$ depicts training curves corresponding to standard- and noncontingent-trained groups. A cursory inspection suggests that the training performance of the noncontingent-trained group was unaffected by its prior 12-h exposure to the context without stimulation. A $2 \times 15$ repeated measures ANOVA that was performed on these data disclosed neither a significant main effect nor a significant context exposure $\times$ trial interaction, but it did disclose a significant trial effect $[F(14,812)=48.5, p<.001]$. Figure $4 \mathrm{c}$ depicts results corresponding to the testing ses- 


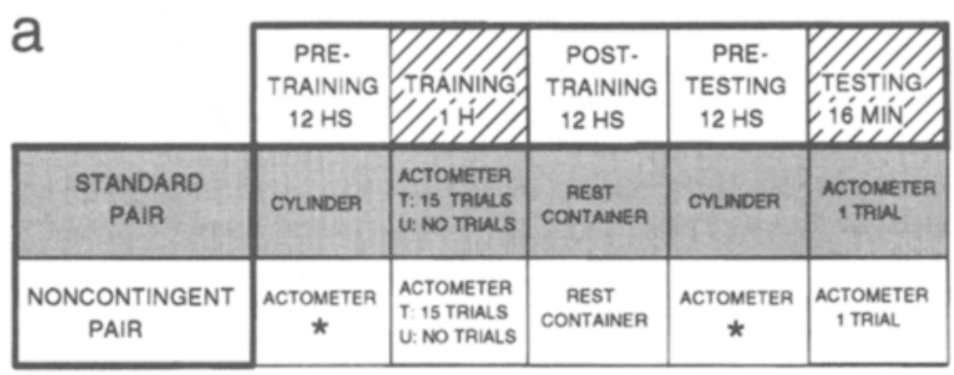

b

TRAINING

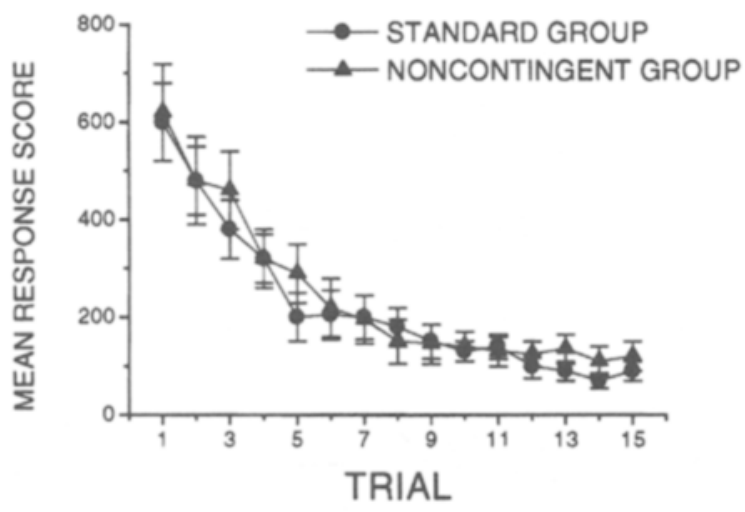

C
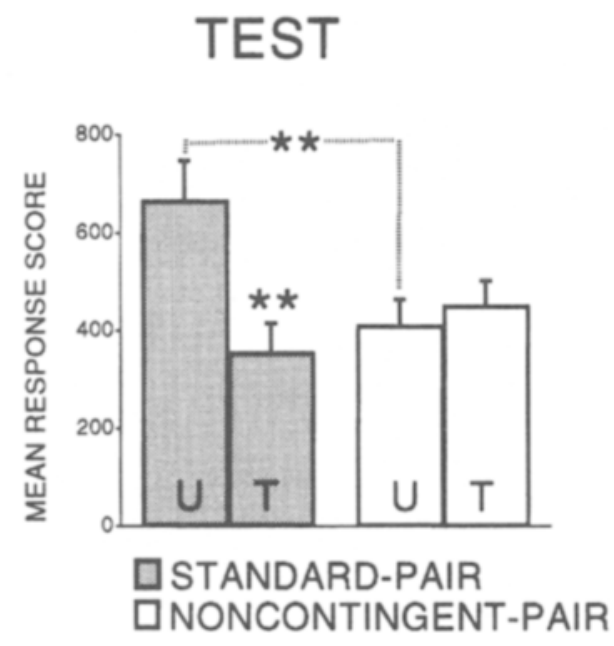

Figure 4. Pretraining and pretesting exposure to the context in the absence of US (Experiment 3). (a) Experimental protocol. The type of container during each phase is pointed. Actometer: the plastic container of each experimental unit (Figure 1a). Cylinder: a plastic transparent cylinder illuminated as the actometer. Rest container: a plastic bowl inside a dimly lit drawer. Asterisks indicate the phase during which the noncontingent group remains in the actometer containers without presentation of the stimulus. The protocol for untrained groups is the same as that for the respective trained ones, but without training trials. (b) Training performance. Black circles stand for the standard-trained group; black triangles, for the noncontingent-trained group ( $n=$ 30). Ordinate: mean response score $\pm S E M$. (c) Testing performance. Gray bars: standard pair of untrained $(U)$ and trained $(T)$ groups that were placed in the actometers only during the training session. White bars: noncontingent pair of untrained $(U)$ and trained $(T)$ groups placed in the actometers both during the training session and during the pretraining and pretesting phases. Other symbols are the same as in Figure 2. 

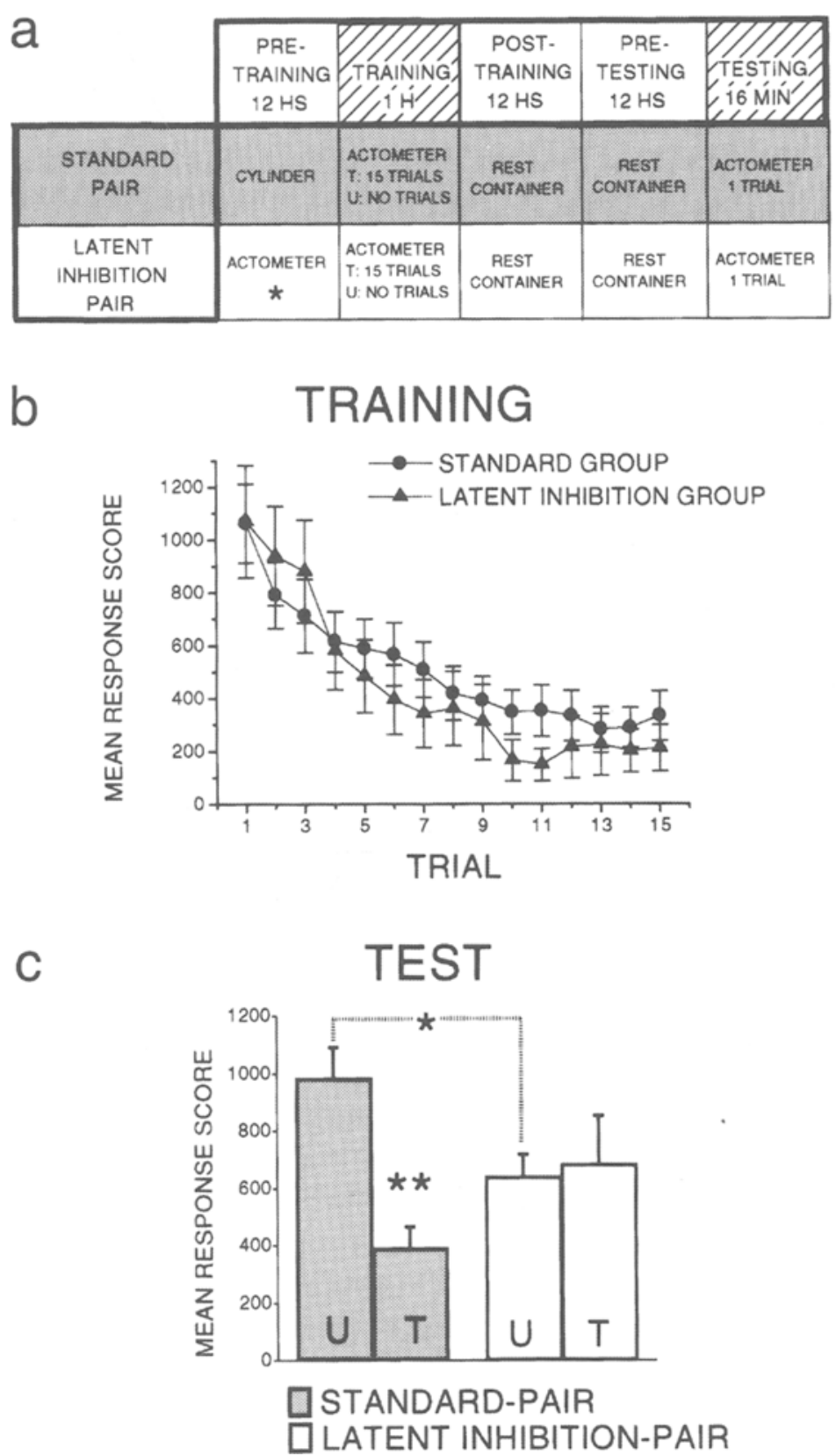

Figure 5. Latent inhibition: pretraining exposure to the context in the absence of US (Experiment 4). (a) Experimental protocol. Symbols are the same as in Figure 4a. (b) Training performance. Symbols are the same as in Figure 4b. (c) Testing performance. Symbols are the same as in Figure 4c.

sion. A two-factor ANOVA on these data revealed a significant main effect of the training $[F(1,116)=8.7, p<$ $.01]$, no significant effect of the context exposure, and a significant interaction between context exposure and training factors $[F=4.4, p<.05]$. Subsequent simple main effects analyses showed a significant difference between the standard groups $[F(1,116)=12.7, p<.01]$, but not between the noncontingent groups, and a significant difference between the untrained groups $[F(1,116)=8.6, p<.01]$.
Concerning untrained groups, results were as expectedthat is, the response level of an untrained group that had been exposed to the actometer containers for two periods of $12 \mathrm{~h}$ (noncontingent-untrained group) was significantly lower than that of a group preexposed for only $1 \mathrm{~h}$ (standarduntrained group) (Figure 4c). Concerning the noncontingenttrained group, the experimental outcome was at odds with the nonassociative prediction and in agreement with the asscciative hypothesis, because the performance of this 


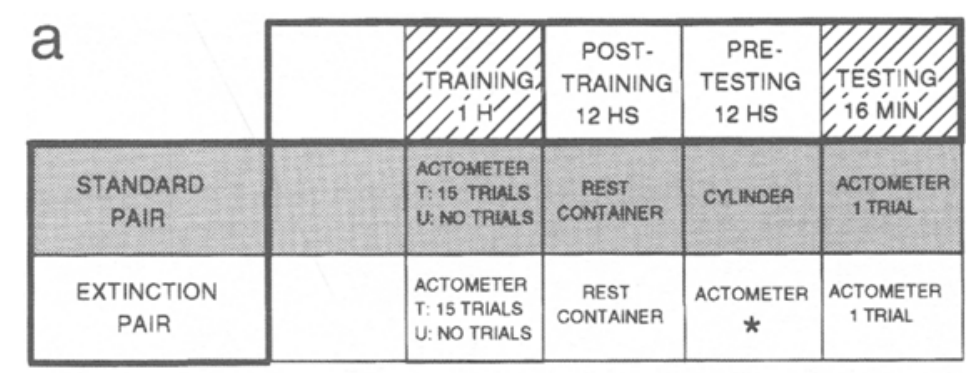

b

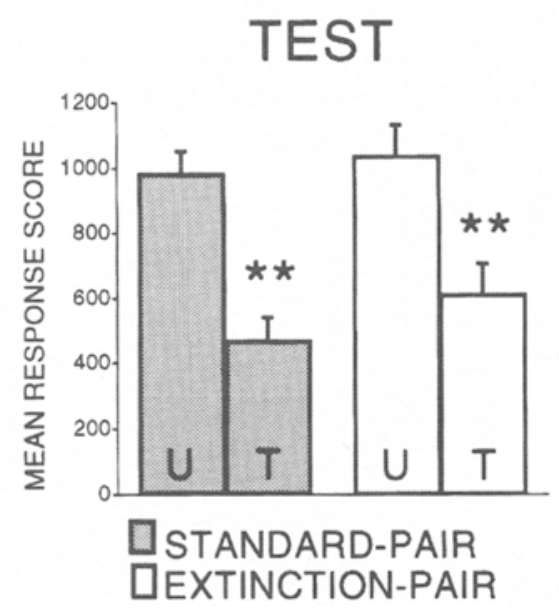

Figure 6. Delayed extinction: pretest exposure to the context in the absence of US (Experiment 5). (a) Experimental protocol. Symbols are the same as in Figure 4a. (b) Testing performance. Symbols are the same as in Figure 4c.

group was not different from that of the respective untrained one. Nevertheless, it should be noted that the results predicted by Wagner's associative theory for this experiment would be a level of response for the noncontingent-trained group higher than that for the standard-trained group. But, as mentioned before, the contextual information acquired by the noncontingent-trained crabs results in a reduction of the escape response to a danger stimulus perceived in that environment. Indeed, the design that was adopted for the experiments in this paper represented an attempt to control for nonspecific sources of changes in reactivity, such as, for example, that originated by the contextual memory effect. Therefore, even though the reactivity of the noncontingent-trained group at test is not higher than that of the standard-trained group, as would be expected according to Wagner's theory, the lack of differences between the noncontingent-trained group and its corresponding untrained one must be interpreted as evidence of a latent inhibition and/or extinction effect.

Now, if LTH is really impaired by noncontingent periods, the question remains of which phase, if not both phases, is responsible for the retention deficit.

An analysis of training results with trained groups showed, unlike that performed on testing results with un- trained groups, no difference between the standard and noncontingent group although the latter was exposed to the context for $12 \mathrm{~h}$ (Figure 4b), which suggests that a 12-h preexposure has no contextual memory effect. Such a disparity is a matter of marked interest, to which we will return later.

\section{EXPERIMENT 4}

If LTH is conceptualized as an associative process, prior exposure to the contextual cues in the absence of the phasic stimulus would operationally correspond to the classical conditioning procedure of latent inhibition (Lubow \& Moore, 1959). Therefore, the purpose of this experiment was to test whether a single 12 -h pretraining phase impairs LTH.

\section{Method}

The experimental design was the same as above, except that the 12-h pretesting phase was omitted, so that all crabs stayed in the rest containers for the usual 24-h intersession interval (posttraining and pretesting phases in Figure $5 \mathrm{a}$ ). Thus, the difference between standard and noncontingent groups consists in the fact that the latter, unlike the former, expanded the pretraining phase in the actometers $(*$ in Figure $5 \mathrm{a}$ ). The four groups ( $n=30$ ) were the standard-trained and the latent-inhibition-trained groups and their respective untrained ones. 


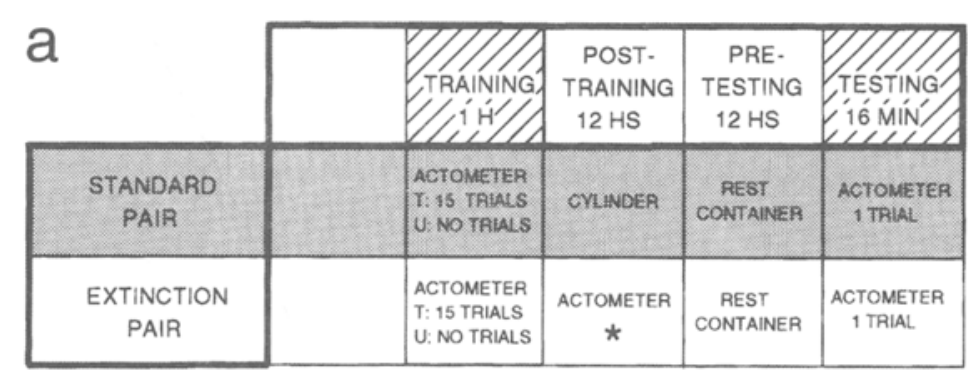

b
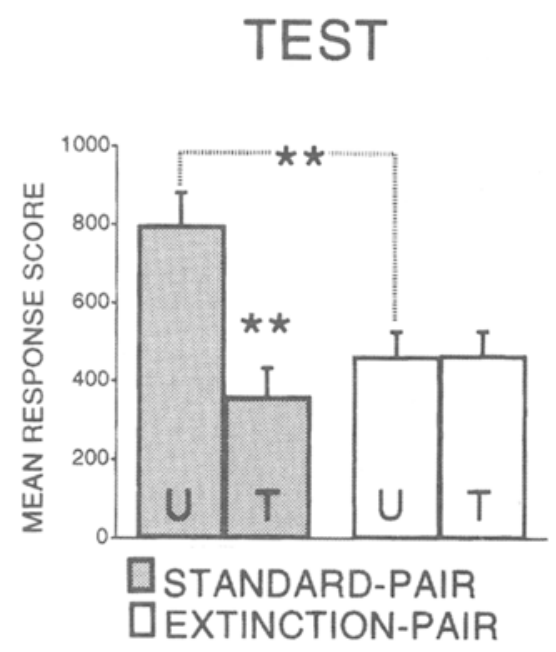

Figure 7. Immediate extinction: posttraining exposure to the context in the absence of US (Experiment 6). (a) Experimental protocol. Symbols are the same as in Figure 3a. (b) Testing performance. Symbols are the same as in Figure 3c.

\section{Results and Discussion}

The training performances of trained groups (Figure $5 \mathrm{~b}$ ) were similar to those exhibited in Figure 4 b. A $2 \times 15$ repeated measures ANOVA that was performed on these data disclosed neither a significant main effect nor a significant context exposure $\times$ trial interaction, but it did disclose a significant trial effect $[F(14,812)=30.0, p<.001]$, thus indicating that the pretraining phase had no attenuating effect on the response level of the latent inhibition-trained group at training.

The results of the testing trial (Figure $5 \mathrm{c}$ ) were basically similar to those of Experiment 3. A two-factor ANOVA on these results revealed a significant effect of training $[F(1,116)=3.9, p<.05]$, no effect of the preexposure to context, and a significant interaction between context exposure and training $[F(1,116)=5.0, p<.05]$. Subsequent simple main effects analyses showed significant differences between the standard groups $[F(1,116)=8.7, p<$ $.01]$ and between the untrained groups $(F=3.9, p<.05)$ but not between the latent-inhibition groups.

Thus, preexposure to the context resulted in a latent inhibition effect and, consequently, in memory impairment. As expected, the presence of a contextual memory effect because of the long exposure was observed. In fact, the escape response level at test of untrained crabs that had been exposed to the actometers for $12 \mathrm{~h}$ (latent-inhibitionuntrained group) was significantly lower than that of untrained animals exposed for $1 \mathrm{~h}$ (standard-untrained group). However, as in Experiment 3, no difference at training was found between a group extensively preexposed (the latentinhibition-trained group) and another one that had not been exposed at all (the standard-trained group).

\section{EXPERIMENT 5}

As noted by Wagner (1976), one powerful prediction that can be derived from his associative model of LTH concerns extinction of the association between the contextual cues and the phasic stimulus. If the contextual stimuli functions as a CS, then posthabituation exposure to the context in the absence of the US should be equivalent to the traditional paradigm of presenting the CS alone and should produce extinction of the association. Therefore, Experiment 5 was designed to test whether a single $12-\mathrm{h}$ pretesting phase impairs LTH.

\section{Method}

The experimental design (Figure 6a) was the same as that of Experiment 3 , except that the 12 -h pretraining phase was omitted, so that all crabs stayed in the rest containers during posttraining, but, 

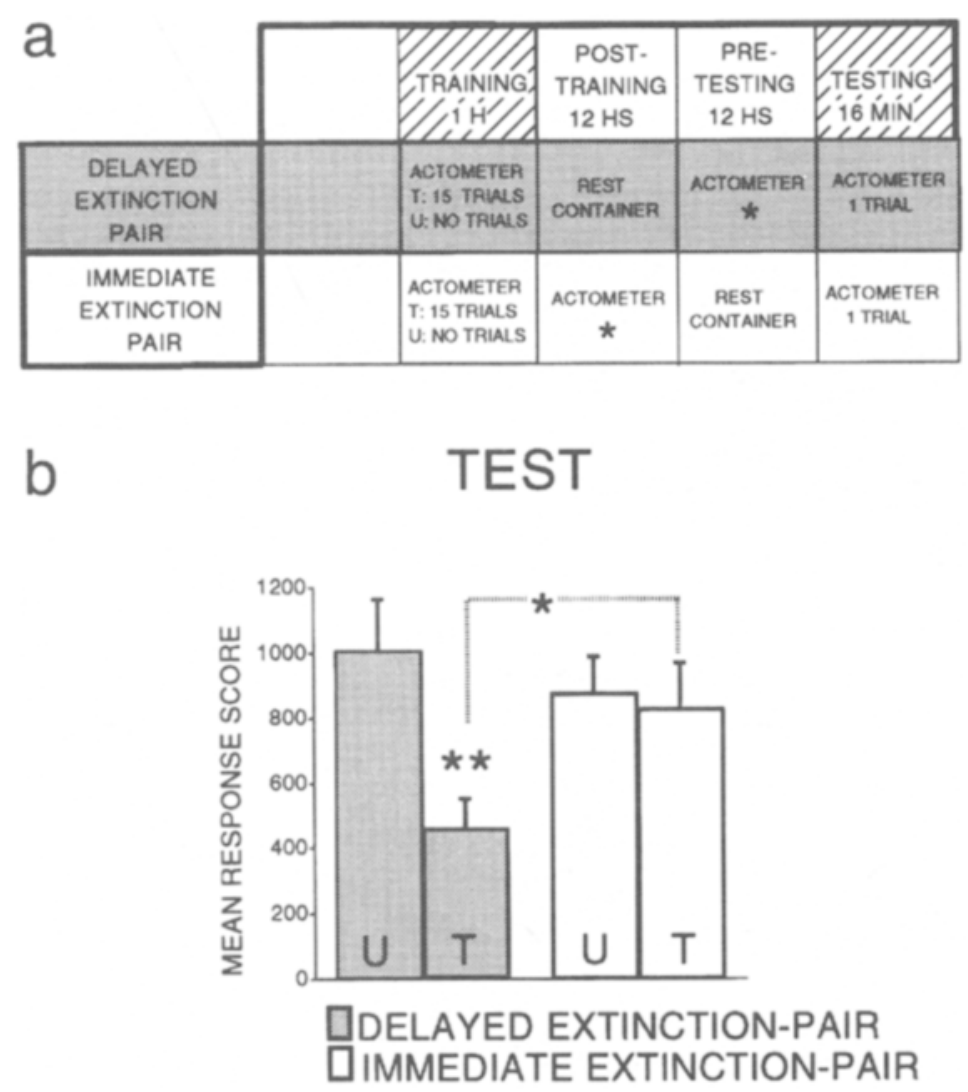

Figure 8. Pretest or posttraining exposure to the context in the absence of US (Experiment 7). (a) Experimental protocol. Symbols are the same as in Figure 4a. (b) Testing performance. Gray bars, trained (T) and untrained (U) delayed extinction groups placed in the actometer during the pretesting phase; white bars, trained $(T)$ and untrained $(U)$ immediate extinction groups placed in the actometer during the posttraining phase. Other symbols are the same as in Figure 4c.

whereas the standard groups spent the pretesting interval in cylinders, the noncontingent groups spent it in actometers (* in Figure $6 \mathrm{a})$. The four groups $(n=30)$ were the standard-trained and the extinction-trained groups and their respective untrained ones.

\section{Results and Discussion}

Figure $6 \mathrm{~b}$ displays results that correspond to the testing session. An ANOVA that was performed on these data revealed a significant effect of training $[F(1,116)=15.0, p<$ $.01]$ but no significant effect of either context exposure or interaction between factors. Subsequent simple main effects analyses exhibited the same pattern of resultsnamely, a significant difference either between the standard groups $[F(1,116)=7.0, p<.01]$ or between the extinction groups $(F=8.0, p<.01)$.

Thus, the results at test were at variance with the predictions. First, the response level at test of the extinctionuntrained group was similar to that of the standard-untrained group - that is, no contextual memory effect was disclosed. Second, no extinction of LTH was observed, which suggests that the lack of retention shown by noncontingenttrained groups in Experiment 3 (Figure $4 \mathrm{c}$ ) could be attributed to latent inhibition alone.
In an experiment previously conducted in our laboratory (Lozada et al., 1990), the extinction groups remained in the actometers for the entire intersession period $(24 \mathrm{~h})$, and a similar result was obtained. A feature common to the present Experiment 5 and the previous experiment of Lozada et al. was the fact that the testing session followed immediately after a long noncontingent exposure to the actometers. Interestingly, when the phasic stimulation is given immediately after a single period of exposure to the context, no context memory effect has been observed (performance of the trained groups at training in Experiments 3 and 4 and that of the untrained groups at testing in the present experiment and in Lozada et al., 1990). Therefore, it seems worth exploring whether, after a context exposure, it is necessary that crabs spend a period in an environment that is different from that of the actometers in order to both disclose a contextual memory effect and disrupt previous context-signal association in trained crabs.

\section{EXPERIMENT 6}

The objective of this experiment was to test whether a posttraining $12-\mathrm{h}$ exposure to the single context impairs 
LTH when it comes immediately after the training and a 12 - $\mathrm{h}$ intervening period is included between the extinction phase and the testing session.

\section{Method}

The experimental design (Figure 7a) was the same as that of Experiment 5, except that the standard groups spent posttraining in cylinders and the extinction groups in actometers, whereas all crabs stayed in the rest containers during the pretesting phase. As in the preceding experiment, the four groups $(n=40)$ were the standardand extinction-trained groups and their respective untrained ones.

\section{Results and Discussion}

Testing results are presented in Figure $7 \mathrm{~b}$. An ANOVA that was performed on these data revealed a significant effect of training $[F(1,156)=9.4, p<.01]$, no significant effect of the context exposure, and a significant effect of interaction $(F=9.6, p<.01)$. Subsequent simple main effects analyses exhibited the same pattern of resultsnamely, significant differences between the standard groups $[F(1,156)=19.0, p<.01]$ but not between the extinction groups and a significant difference between the untrained groups $(F=11.0, p<.01)$. Extremely close results were obtained in a replication of this experiment ( $n=30$, per group).

This result is in agreement with what is perhaps the most striking prediction of Wagner's theory-namely, extinction of the association between environmental cues and the habituating stimulus. However, taking into account this result, that of Experiment 5, and that previously reported by Lozada et al. (1990), the extinction procedure seems to disrupt LTH only when a period of exposure to an environment that is different from the experimental context is interpolated between the noncontingent phase and the test. Because it is a quite unexpected finding and because no definitive conclusion can be drawn by a betweenexperiment comparison, the following experiment aimed to explore this issue further.

\section{EXPERIMENT 7}

The purpose here was to assess, in a single experiment, the extinction effect of a 12-h exposure to context on LTH, when the exposure follows the training (and a 12-h period in a different environment precedes the test) or when it is given $12 \mathrm{~h}$ after the training (i.e., immediately preceding the test).

\section{Method}

The experimental design (Figure 8a) was the same as that of Experiments 5 and 6 , except that no standard pair was included. One of the pairs spent the posttraining phase in the cylinders and the pretesting phase in the actometers, whereas the other pair spent the posttraining in the actometers and the pretesting phase in the cylinders. The four groups $(n=30)$ were the immediate-extinction-trained group and the delayed-extinction-trained group and their respective untrained ones.

\section{Results and Discussion}

An ANOVA that was performed on the testing data revealed a significant effect of training $[F(1,116)=5.4, p<$
.05], no significant effect of the period of context exposure, and a significant effect of interaction $(F=4.0, p<$ .05 ). Subsequent simple main effects analyses exhibited the same pattern of results - that is, a significant difference between the delayed-extinction groups that received the long exposure to the actometers during the pretesting phase $[F(1,116)=9.1, p<.01]$, but no difference between the immediate-extinction groups that remained in the actometer during the posttraining phase. It is worthwhile to notice that an analogous analysis on the simple main effects of the factor period of context exposure revealed a significant difference between trained groups $(F=5.4$, $p<.05$ ).

The result of this experiment confirms the observation, drawn from Experiments 5 and 6, that an effect of extinction takes place when the crabs are exposed to the context alone; however, such an effect occurs only if an intervening period, spent in a different environment, is present before the test.

\section{GENERAL DISCUSSION}

The results of the present paper show that context shift as well as latent inhibition and extinction treatments impair retention of the habituated response, which suggests that Chasmagnathus LTH can be largely accounted for by Wagner's associative theory of habituation.

The failure of Chasmagnathus to exhibit habituation when tested in a context different from that of training might be considered to be due to the sensitizing effect of the different context, rather than to Wagner's proposed associative process. This proposal has been made by Hall and Channell (1985) for experimental results with rats. However, such an interpretation is not tenable for the present results because, as mentioned before, the experimental design used in the present study was adopted to control for such kinds of nonspecific sources of changes in reactivity level. In fact, there was no difference between the untrained groups in Experiments 1 and 2 (Figures $2 b$ [neither in $\mathrm{N}$ nor in $\mathrm{S}$ ] and $3 \mathrm{~b}$, respectively), and this analogous reactivity of untrained groups could be not explained by a ceiling effect, as scores twice as high are obtained rou-. tinely in our laboratory using stimuli other than an opaque screen moved horizontally (Lozada et al., 1990).

The main outcome of this paper is at variance with the majority of empirical studies of LTH, inasmuch as they have failed to find any evidence for an effect of context shift (for a review, see Mackintosh, 1987; see, e.g., Schaafsma, Packer, \& Siddle, 1989). However, a negative result does not explain away contextual association. The absence of an effect may be due to any number of reasons. For example, the environmental change may not have been discriminable by the subject (Marlin \& Miller, 1981). Alternatively, the chosen parameter of the background may have been an irrelevant cue in the contextual memory (Davis \& File, 1984). Lastly, it is very possible that not all types of LTH or habituation in different species will ultimately be explained by the same theoretical mechanism. 
Association to diffuse background (contextual) stimuli has recently been reported in the marine mollusk Hemissenda crassicornis (Rogers, Schiller, \& Matzel, 1996). Moreover, context-US association as a determinant of sensitization - the other classically nonassociative form of learning - has been described in invertebrates (Balaban \& Bravarenko, 1993; Colwill, Absher, \& Roberts, 1988). However, to the best of our knowledge, context-US association as a determinant of LTH has been demonstrated only in the present paper.

These and previous experiments also indicate that the contextual memory effect induced by exposure to the context alone depends on an intervening period. When such an interval was included, the results from Tomsic et al. (1993), Pedreira et al. (1995), Pedreira et al. (1996), and Saraco and Maldonado (1995) showed that the exposure to the single context induced a clear-cut reduction of the escape response, in comparison with that of a group without exposure. Coincidentally, the results from Experiments 3, 4, and 6 of the present paper exhibited a similar tendency in the comparison of the performance at test of an untrained group that had been exposed to the single context for a long period with that of another which had also been preexposed to the actometer, but for a shorter period. In contrast, when no intervening period was included - that is, when the phasic stimulation was given immediately after a single period of noncontingent exposure - groups exhibited a similar level of responding to the danger stimulus (performances of trained groups at training in Experiments 3 and 4 and those of untrained groups at testing in Experiment 5 and in Lozada et al., 1990).

In summary, long-term exposure of Chasmagnathus to the context in the absence of a phasic stimulation has a double effect. First, the response level to phasic stimulation is attenuated. Second, LTH is impaired when the noncontingent exposure to the context precedes the habituation training (latent inhibition; Experiments 3 and 4) or when the former comes after the latter (extinction; Experiment 6). However, such a double effect takes place only when an intervening period in a different environment is inserted between exposure to the single context and phasic stimulation at test. This is, one has to admit, an unexpected and intriguing result that is not predicted by Wagner's theory or by other memory theories. It seems that these crabs require some time away from the context, or need to be exposed to an alternate context, to consolidate contextual information. Perhaps, for animals such as Chasmagnathus that live in individual burrows but forage around on a daily basis, it could be a mechanism that helps the animal to recall the environment of its burrow without interference from other recently visited contexts.

After all, most theories of learning and memory were based on, or at least strongly influenced by, experimentation performed with laboratory-reared animals. Comparatively, not very much is known about how memory pro- cessing takes place in wild animals or about whether different species of wild animals acquire and store information in the same way.

\section{REFERENCES}

Applewhite, B. P., Gardner, F. T., \& Lapan, E. (1969). Physiology of habituation learning in a protozoan. Transactions of the New York Academy of Sciences, 31, 842-849.

Balaban, P. M., \& Bravarenko, N. (1993). Long term sensitization and environmental conditioning in terrestrial snails. Experimental Brain Research, 96, 487-493.

BrunNER, D., \& Maldonado, H. (1988). Habituation in the crab Chasmagnathus granulatus: Effect of morphine and naloxone. Journal of Comparative Physiology A, 162, 687-694.

Colwill, R. M., Absher, R. A., \& Roberts, M. L. (1988). Context-US learning in Aplysia californica. Journal of Neuroscience, 8, 4434-4439.

Davis, M., \& FILE, S. E. (1984). Intrinsic and extrinsic mechanisms of habituation and sensitization: Implications for the design and analysis of experiments. In H. V. S. Peeke \& L. Petrinovich (Eds.), Habituation, sensitization and behavior (pp. 287-323). New York: Academic Press.

Groves, P. M., \& Thompson, R. F. (1970). Habituation: A dual-process theory. Psychological Review, 77, 419-450.

Hall, G., \& Channell, S. (1985). Differential effects of contextual change on latent inhibition and on the habituation of an orienting response. Journal of Experimental Psychology: Animal Behavior Processes, 11, 470-481.

Korn, J. H., \& MoYer, K. E. (1966). Habituation of the startle response and of heart rate in rat. Canadian Journal of Psychology, 20, 183-190.

Lubow, R. E., \& MoOrE, A. U. (1959). Latent inhibition: Effect of non reinforced preexposure to the conditioned stimulus. Journal of Comparative Physiology \& Psychology, 52, 415-419.

LozAdA, M. (1993). Habituación a largo término y especificidad del estímulo en el cangrejo Chasmagnathus granulatus. Unpublished doctoral dissertation, University of Buenos Aires.

Lozada, M., Romano, A., \& Maldonado, H. (1990). Long-term habituation to a danger stimulus in the crab Chasmagnathus granulatus. Physiology \& Behavior, 47, 35-41.

MaCKINTOSH, N. J. (1987). Neurobiology psychology and habituation. Behavior Research \& Therapy, 25, 81-97.

Maldonado, H., Romano, A., \& Tomsic, D. (1997). Long-term habituation (LTH) in the crab Chasmagnathus: A model for behavioral and mechanistic studies of memory. Brazilian Journal of Medical \& Biological Research, 30, 813-826.

MARLIN, N. A., \& MilleR, R. R. (1981). Association to contextual stimuli as a determinant of long term habituation. Journal of Experimental Psychology: Animal Behavior Processes, 7, 313-333.

Pedreira, M. E., Dimant, B., \& Maldonado, H. (1996). Inhibitors of protein and RNA synthesis block context memory and long-term habituation in the crab Chasmagnathus. Pharmacology, Biochemistry \& Behavior, 54, 611-617.

Pedreira, M. E., Dimant, B., Tomsic, D., Quesada-Allue, L. A., \& Maldonado, H. (1995). Cycloheximide inhibits context memory and long-term habituation in the crab Chasmagnathus. Pharmacology, Biochemistry \& Behavior, 52, 385-395.

Pedreira, M. E., Romano, A., Tomsic, D., Lozada, M., \& MaldonADO, H. (1998). Massed and spaced training build up different components of long-term habituation in the crab Chasmagnathus. Animal Learning \& Behavior, 26, 34-45.

Peeke, H. V. S., \& Veno, A. (1976). Stimulus specificity of habituated aggression in the three-spined sticklebacks Gasterosteus acualeatus. Zeitschrift für Tierpsychologi, 40, 53-58.

Pereyra, P., de la Iglesia, H., \& Maldonado, H. (1996). Training to testing intervals different from 24 hours impair habituation in the crab Chasmagnathus. Physiology \& Behavior, 59, 19-25.

Rakitin, A., Tomsic, D., \& Maldonado, H. (1991). Habituation and 
sensitization to a electrical shock in the crab Chasmagnathus: Effect of background illumination. Physiology \& Behavior, 50, 477-487.

REsCORLA, R. A. (1988). Behavioral studies of Pavlovian conditioning. Review of Neuroscience, 1, 329-352.

RoGers, R. F., SChILler, K. M., \& MATZEL, L. D. (1996). Chemosensorybased contextual conditioning in Hermissenda crassicornis. Animal Learning \& Behavior, 24, 28-37.

Romano, A., Lozada, M., \& Maldonado, H. (1991). Nonhabituation processes affect stimulus specificity response habituation in the crab Chasmagnathus granulatus. Behavioral Neuroscience, 105, 542-552.

Saraco, M., \& Maldonado, H. (1995). Ethanol affects context memory and long-term habituation in the crab Chasmagnathus. Pharmacology, Biochemistry \& Behavior, 51, 223-229.

Schaafsma, M. F., Packer, J. S., \& Siddle, D. A. T. (1989). The effect of context change and long term habituation of the skin conductance response to a signal and nonsignal stimuli in humans. Biological Psychology, 29, 181-191.

SPEAR, N. E. (1973). Retrieval of memory in animals. Psychological Review, 80, 163-175.

SPEAR, N. E. (1978). The processing of memories: Forgetting and retention. Hillsdale, NJ: Erlbaum.

Tomsic, D., Dimant, B., \& Maldonado, H. (1996). Age-related deficits of long-term memory in the crab Chasmagnathus. Journal of Comparative Physiology A, 178, 139-146.

Tomsic, D., Maldonado, H., \& Rakitin, A. (1991). Morphine and
GABA: Effects on perception, escape response and long-term habituation to a danger stimulus in the crab Chasmagnathus. Brain Research Bulletin, 26, 699-706.

Tomsic, D., Massonl, V., \& Maldonado, H. (1993). Habituation to a danger stimulus in two semiterrestrial crabs: Ontogenic, ecological and opioid modulation correlates. Journal of Comparative Physiology $A, 173,621-633$.

WAGNER, A. R. (1976). Priming in STEM: An information processing mechanism for self-generated or retrieval-generated depression in performance. In T. J. Tighe \& R. N. Leaton (Eds.), Habituation: Perspectives from child development, animal behavior and neurophysiology (pp. 95-128). Hillsdale, NJ: Erlbaum.

WAGNER, A. R. (1978). Expectancies and the priming of STM. In S. H. Hulse, H. Fowler, \& W. R. Honing (Eds.), Cognitive processes in animal behavior (pp. 177-209). Hillsdale, NJ: Erlbaum.

WAGNer, A. R. (1979). Habiuation and memory. In A. Dickinson \& R. A. Boakes, Mechanisms of learning and motivation (pp. 53-82). Hillsdale, NJ: Erlbaum.

WhITLOW, J. W., \& WAGNER, A. R. (1984). Memory and habituation. In H. V. S. Peeke \& L. Petrinovich (Eds.), Habituation, sensitization and behavior (pp. 103-153). New York: Academic Press.

(Manuscript received September 5, 1996; revision accepted for publication August 23, 1997.) 